\title{
Improving the RF Performance of Carbon Nanotube Field Effect Transistor
}

\author{
S. Hamieh \\ Faculty of Sciences I, Lebanese University, Beirut, Lebanon \\ Correspondence should be addressed to S. Hamieh, hamiehs@yahoo.fr \\ Received 27 September 2011; Accepted 9 February 2012 \\ Academic Editor: Teng Li \\ Copyright () 2012 S. Hamieh. This is an open access article distributed under the Creative Commons Attribution License, which \\ permits unrestricted use, distribution, and reproduction in any medium, provided the original work is properly cited. \\ Compact model of single-walled semiconducting carbon nanotube field-effect transistors (CNTFETs) implementing the calcu- \\ lation of energy conduction subband minima under VHDLAMS simulator is used to explore the high-frequency performance \\ potential of CNTFET. The cutoff frequency expected for a MOSFET-like CNTFET is well below the performance limit, due to \\ the large parasitic capacitance between electrodes. We show that using an array of parallel nanotubes as the transistor channel \\ combined in a finger geometry to produce a single transistor significantly reduces the parasitic capacitance per tube and, thereby, \\ improves high-frequency performance.
}

\section{Introduction}

Carbon nanotubes belong to the fullerenes family and are sheets of graphite rolled in the shape of a tube. Depending on the direction in which the nanotubes are rolled (chirality), they can be either metallic or semiconducting [1]. Semiconducting nanotubes have been used in high-performance transistors where the channel is the nanotube itself. Since the first demonstration of carbon nanotube fieldeffect transistors (CNTFETs) in 1998 [2, 3], there has been an immense research concerning the electrical properties and the physical understanding of CNTFET $[1,4,5]$. The manufacturing of CNTFET processes progress continually: one of the most advanced is the chemical vapor deposition technique (CVD). The CNTFET can be characterized by high carrier mobility, low leakage current, important on state current relatively to the applied voltages, and low inverse subthreshold slope. These properties allow us to consider the design of high-speed and high-performance electronic circuits. High-performance CNTFET operating close to the ballistic limit has been reported in [6-8]. However, due to the large size of the probe pads relative to the CNT device, the parasitic capacitance of the pads can dominate the measured frequency response and inhibit measurement of the intrinsic response of CNT transistors. Moreover, the low current drive and high-input/output impedance of single CNTFET make it difficult to perform direct measurements of high-frequency electrical properties using instrumentation based on a reference impedance of $50 \Omega$. In order to make a direct measurement of a recognized high-frequency figure of merit, such as $f_{T}$ small signal $S$-parameter measurements were achieved by a newly developed multiple-channel CNTFET structure whose output impedance is much lower than the usual single-channel CNTFET and a deembedding scheme that removes existing errors in measured $S$-parameters [9]. The authors of [9] measure $f_{T}=10.3 \mathrm{GHz}$ after de-embedding. More recently, using one individual $100 \mu \mathrm{m}$ long single-walled carbon nanotube, 100 individual nanotube top-gated field effect transistors are combined in a finger geometry to produce a single transistor with a cutoff frequency (after deembedding parasitic capacitance of the finger structure) of $7.6 \mathrm{GHz}$; before deembedding the cutoff frequency is $0.2 \mathrm{GHz}$ [10].

In this work, we use compact model, in a quasi-static approach simulations to examine the high-frequency performance potential for a state-of-the-art CNTFET. Using an array of parallel nanotubes as the transistor channel combined in a finger geometry to produce a single transistor significantly reduces the parasitic capacitance per tube and, thereby, improves high-frequency performance. The results presented here should prove useful for understanding and 
optimizing high-frequency characteristics of CNTFET and assessing the potential of CNTFET for nanoelectronic RF applications.

This paper is organized as follows. In Section 2, the compact model is presented. In Section 3, the results of our model using VHDL-AMS simulator in the quasistatic approach are shown and discussed. In Section 4 a new architecture is proposed and the results of the proposed architecture are presented. The conclusions are summarized in Section 5.

\section{Approach}

A simple model for ballistic nanotransitors is described in $[11,12]$. For details about this model, we refer the reader to [13-15]. In this model, the gate voltage $V_{\mathrm{gs}}$ induces charge in the CNTFET channel $Q_{\mathrm{cnt}}$. It also modulates the top of the energy band between the source and the drain by an amount $V_{\text {cnt }}$. As the source-drain barrier is lowered, current flows between the source and the drain. The electrons coming from the source fill $+k$ states and the electrons coming from the drain fill up the $-k$ states. The control potential $V_{\text {cnt }}$ is computed self consistently using

$$
V_{\mathrm{cnt}}=\frac{\mathrm{LC}_{I} V_{\mathrm{gi}}+C_{\mathrm{SE}} V_{\mathrm{si}}+C_{\mathrm{DE}} V_{\mathrm{di}}-Q_{\mathrm{cnt}}}{\mathrm{LC}_{I}+C_{\mathrm{DE}}+C_{\mathrm{SE}}},
$$

where $V_{\text {gi }}, V_{\text {si }}$, and $V_{\text {di }}$ are the intrinsic gate, source, and drain potential respectively. $C_{\mathrm{SE}}$, and $C_{\mathrm{DE}}$ are the contact capacitance of the source and drain, $C_{I}$, is the gate oxide capacitance (see Table 1), $Q_{\mathrm{cnt}}$ depends on the number of carriers in the channel $n_{\text {cnt }}$ which is the sum of the energy subband contributions, and $L$ is the nanotube length. The drain current equation is derived from the Landauer formula which describes ballistic transport with ideal contacts:

$$
\begin{aligned}
I=\frac{4 e k_{B} T}{h} \sum_{p} & {\left[\ln \left(1+\exp \frac{-V_{\mathrm{si}}-\Delta_{p}+V_{\mathrm{cnt}}}{k_{B} T}\right)\right.} \\
& \left.-\ln \left(1+\exp \frac{-V_{\mathrm{di}}-\Delta_{p}+V_{\mathrm{cnt}}}{k_{B} T}\right)\right],
\end{aligned}
$$

where $\Delta p$ is the minima of the $p$ th energy subband. The subbands minima can be calculated using zone folding method of the graphene electron dispersion:

$$
\epsilon_{\nu}(\mathbf{k})=\epsilon_{\text {graphene }}\left(|\mathbf{k}| \frac{\mathbf{K}_{\mathbf{2}}}{\left|\mathbf{K}_{\mathbf{2}}\right|}+\nu \mathbf{K}_{\mathbf{1}}\right)
$$

with

$$
\begin{gathered}
v=-\left(\frac{N}{2}+1\right), \ldots, \frac{N}{2}, \\
-\frac{\pi}{|\mathbf{T}|}<|\mathbf{k}|<\frac{\pi}{|\mathbf{T}|},
\end{gathered}
$$

where $|\mathbf{T}|$ is the unit vector for the carbon nanotube (CNT), $N$ is the number of hexagons in CNT unit cell, and $\mathbf{K}_{\mathbf{1}}$ and $\mathbf{K}_{\mathbf{2}}$ define the CNT reciprocal unit cell [16].
TABLE 1: CNTFET parameters (see text for discussion about the used values of the parameters).

\begin{tabular}{llcc}
\hline Parameter & Name & Value & Unit \\
\hline$n$ & Helicity parameter & 19 & \\
$m$ & Helicity parameter & 0 & \\
$d$ & Diameter & $1.49 \times 10^{-9}$ & $\mathrm{~m}$ \\
$T$ & Temperature & 300 & $\mathrm{~K}$ \\
$R_{d}$ & Drain contact resistance & $17 \times 10^{3}$ & $\Omega$ \\
$R_{s}$ & Source contact resistance & $23 \times 10^{3}$ & $\Omega$ \\
$R_{g}$ & Gate contact resistance & 10 & $\Omega$ \\
$L$ & Nanotube length & $100 \times 10^{-9}$ & $\mathrm{~m}$ \\
$V_{\mathrm{FB}}$ & Flat band potential & $-40 \times 10^{-3}$ & $\mathrm{~V}$ \\
$C_{I}$ & Gate oxide capacitance & $100 \times 10^{-12}$ & $\mathrm{~F} / \mathrm{m}$ \\
$C_{\mathrm{DE}}$ & Drain capacitance & $0.1 \times 10^{-18}$ & $\mathrm{~F}$ \\
$C_{\mathrm{SE}}$ & Source capacitance & $0.1 \times 10^{-18}$ & $\mathrm{~F}$ \\
\hline
\end{tabular}

\section{Quasistatic Approach and Results of Simulation}

In the quasistatic approach to transistor modeling, dynamic behavior is predicted by employing static equations for charge (or carrier density) and transport current, but with static voltages replaced by their time-dependent counterparts:

$$
\begin{gathered}
Q\left(V_{G}, V_{D}, V_{S}\right) \longrightarrow Q\left(V_{G}(t), V_{D}(t), V_{S}\right)(t), \\
I\left(V_{G}, V_{D}, V_{S}\right) \longrightarrow I\left(V_{G}(t), V_{D}(t), V_{S}\right)(t),
\end{gathered}
$$

where $Q$ is charge, $I$ is current, $t$ is time, and $V_{G}, V_{D}$, and $V_{S}$ are the gate, drain, and source voltages, respectively. Employing the model presented in the last section with the quasistatic assumption, one can determine the ac behavior of CNTFET. The standard procedure is to assume sinusoidal excitation, write each time-dependent quantity in (5) in terms of static (dc or bias) and dynamic (ac, or small-signal) parts, for example, $V(t)=V_{c}+V_{a} \exp (j w t)$ where $V_{c}$ refers to the dc value and $V_{a}$ refers to the complex ac amplitude, and then make simulation to solve the equations for the ac terminal currents in terms of the ac terminal voltages.

3.1. VHDL-AMS Implementation. The MOSFET-like CNTFET compact model introduced in last section using the quasistatic approach is implemented with VHDL-AMS simulation tool. The main quantities used in the model are the control potential $V_{\mathrm{cnt}}$, the subbands energy level $\Delta p$, and the source (drain) Fermi level $\mu_{S(D)}$. A maximum of 12 intrinsic parameters are necessary as input of this model: the chirality vector $(n, m)$, the diameter, the flatband voltage $V_{\mathrm{FB}}$, the contact resistances $\left(R_{s}, R_{d}, R_{g}\right)$, the contact drain (source) capacitance $C_{\mathrm{DE}}\left(C_{\mathrm{SE}}\right)$, and the gate oxide capacitance per unit length $C_{I}$. (The values of the parameters shown in Table 1 are estimated under the assumption that the compact model results for $I-V$ characteristics should be compatible with the results of the model based on Boltzmann transport equation.) The value of $C_{I}$ can be calculated from, $C_{I}=2 \pi \epsilon_{0} \epsilon_{o x} /((2 h+d) / d)$, for a nanotube surrounded by 


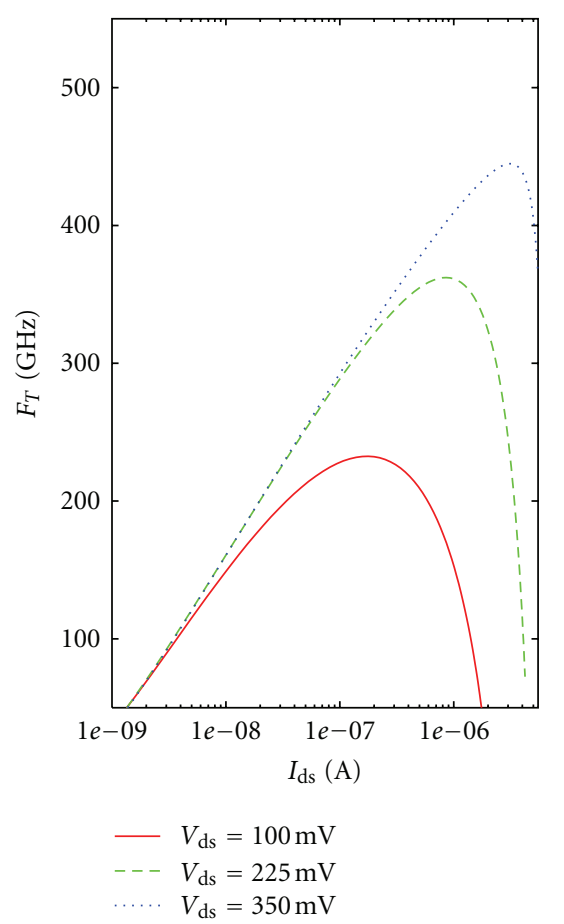

Figure 1: Intrinsic $f_{T}$ as function of $I_{\mathrm{ds}}$ for $V_{\mathrm{ds}}=100 \mathrm{mV}$ (solid line), $V_{\mathrm{ds}}=225 \mathrm{mV}$ (dotted line), and $V_{\mathrm{ds}}=350 \mathrm{mV}$ (dashed line).

a coaxial gate, both separated by an oxide of thickness $h$ with permittivity $\epsilon_{o x}$. The device parameters used in the simulation for the $I-V$ characteristics are summarized in Table 1. In Figure 1, we show the results of simulation based on our model for the intrinsic $F_{T}$ as function of $I_{\mathrm{ds}}$ for $V_{\mathrm{ds}}=100 \mathrm{mV}$ (solid line), $V_{\mathrm{ds}}=225 \mathrm{mV}$ (dotted line), and $V_{\mathrm{ds}}=350 \mathrm{mV}$ (dashed line). The variation of $F_{T}$ with $I_{\mathrm{ds}}$, which is apparent in Figure 1, can be divided into three regions. region $I$ is the low current region where $F_{T}$ decreases as $I_{\mathrm{ds}}$ decreases, region $I I$ is midcurrent region where $F_{T}$ is approximately constant, and region $I I I$ is the high-current region where $F_{T}$ decreases as $I_{\mathrm{ds}}$ increases. The reasons for this behavior of $F_{T}$ with $I_{\mathrm{ds}}$ can be appreciated by plotting $I_{\mathrm{ds}}$ as function of $V_{\mathrm{gs}}$ at constant $V_{\mathrm{ds}}$ where $g_{m}=\partial I_{\mathrm{ds}} /\left.\partial V_{\mathrm{gs}}\right|_{V_{\mathrm{ds}}}$ is zero in the high-current region. The results of the simulation are very promising: the maximum obtained value of $f_{T} \sim 450 \mathrm{GHz}$. However, if the extrinsic parasitic capacitance was included the RF performance of the carbon nanotube transistor will be reduced significantly. This will be our goal in the next section where we propose a new architecture to improve the performance of the CNTFET in the RF regime.

\section{Multifinger Multitube Field-Effect Transistors}

In this section, a new architecture "multifinger multitube field-effect transistor" (MMFET) is proposed that expected to increase the performance of the nanotube in high-frequency applications [10]. In the proposed architecture, using an array of parallel nanotubes as the transistor channel

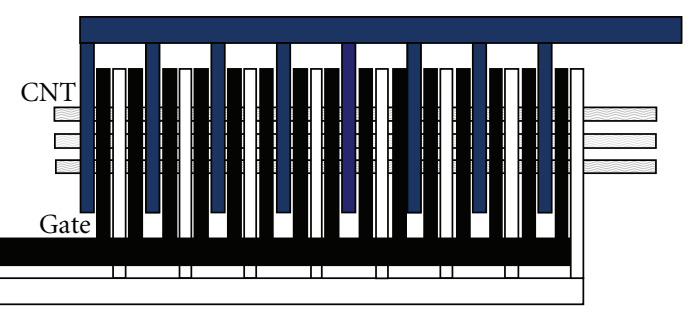

FIGURE 2: Schematic indication of MMFET device geometry (not to scale). In total, there are 16 tubes and 25 fingers (gates) in our design.

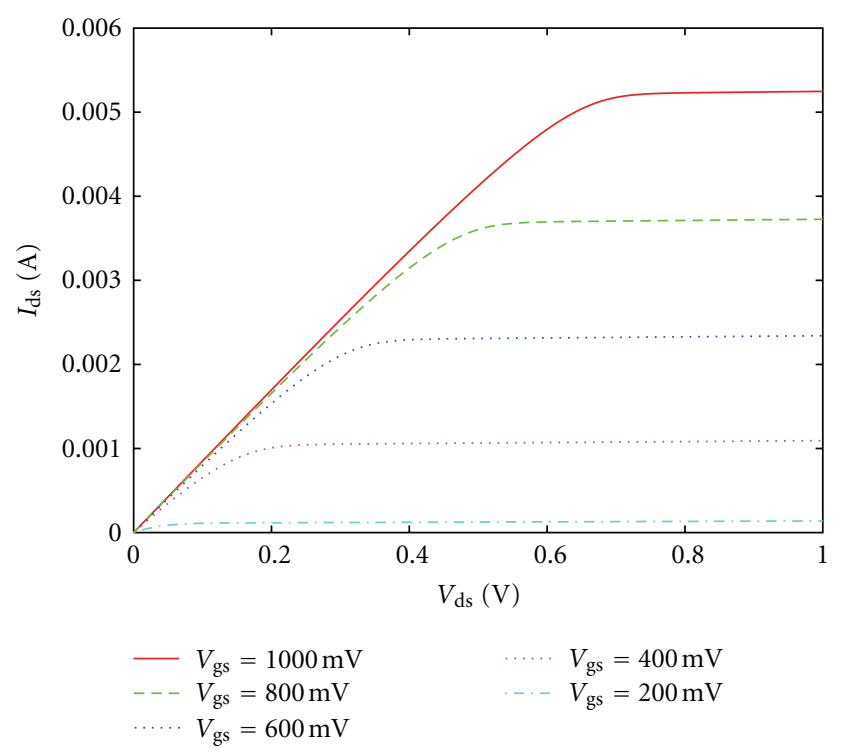

FIGURE 3: $I_{\mathrm{ds}}-V_{\mathrm{ds}}$ for the $(19,0)$ CNTFET (color online).

combined in a finger geometry to produce a single transistor significantly reduces the parasitic capacitance per tube and, thereby, improves high-frequency performance. Shown in Figure 2 a schematic indication of the MMFET geometry (not to scale). In total, there are 16 tubes and 25 fingers (gate) in our design. The distance between two nanotubes is taken to be $2 d$ [17]. In the next two subsection we will study the DC performance and dispersion of this new architecture, and in the last two subsections, we will study the RF performance and applications of such architecture in RF circuits like ring oscillator.

4.1. DC Performance of MMFET. Figure 3 shows the simulated $I_{\mathrm{ds}}-V_{\mathrm{ds}}$ characteristic for constant $V_{\mathrm{gs}}$ (see figure) at room temperature of our device. The device parameters used in the simulation are summarized in Table 1. The simulation has taken into account the series resistances. As one can expect, the current found for MMFET is $400 \times$ the single CNTFET current for the same characteristics.

4.2. Dispersion. Many physical parameters values vary in a wide range when nanotubes are manufactured. The most important impact is due to diameter dispersion. Here we will study the impact of the dispersion on the obtained diameters 


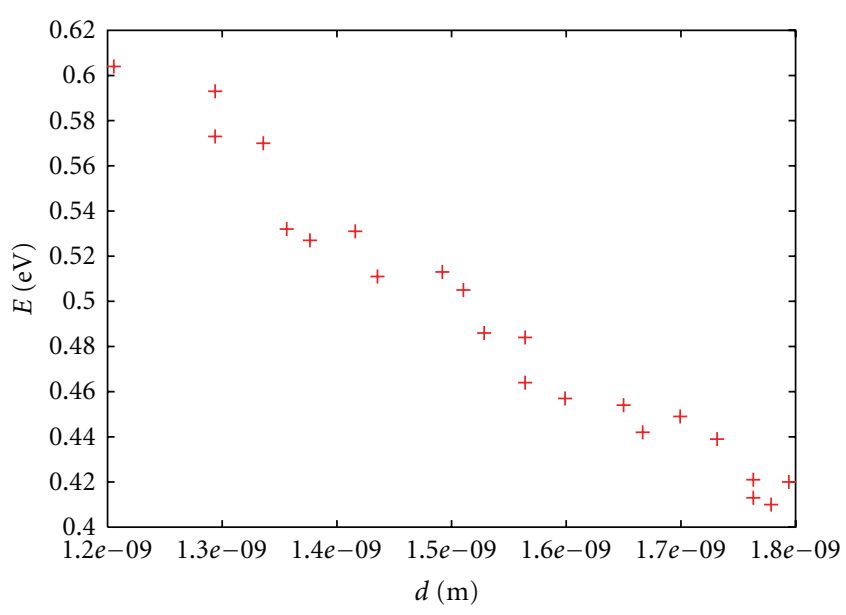

Figure 4: Minimum of the nanotube energy subband versus tube diameter.

based on chirality vectors. The simulation is done using the following steps.

(1) Find all chirality vectors $(m, n)$ which produce a diameter between $1.2 \mathrm{~nm}$ and $1.8 \mathrm{~nm}$.

(2) Eliminate those vectors that give a metallic nanotube. 22 chirality vectors are found after elimination.

(3) Generate a uniform random number to pick up 16 chirality vectors out of the 22 chirality vectors. (Note that, the 16 chirality vectors correspond to the 16 nanotubes used in our simulation in the new architecture.)

(4) Generate another uniform random numbers for each trial.

In Figure 4, we show the minimum sub-band energy of the nanotube versus tube diameter for each chirality vector. The impact of the diameter dispersion on the $I_{\mathrm{ds}}-V_{\mathrm{gs}}$ characteristic at $V_{\mathrm{ds}}=800 \mathrm{mV}$ is shown in Figure 5. According to the results shown in Figure 5, we can conclude that the $I_{\mathrm{on}} / I_{\text {off }}$ ratio is very sensitive to diameter spreading. To see the distribution of $I_{\mathrm{ds}}$ for fixed $V_{\mathrm{ds}}=0.8 \mathrm{~V}$, we show the current versus number of events that fall within $I_{\mathrm{ds}}$ and $I_{\mathrm{ds}} \pm \Delta I_{\mathrm{ds}}$ with $\Delta I_{\mathrm{ds}}=0.2 \mathrm{~mA}$. This distribution, as one can expect, can be compared to gaussian distribution with, in this case, a mean value $I_{\mathrm{ds}}^{\text {mean }} \sim 3.4 \mathrm{~mA}$ and $\sigma \sim 0.21 \mathrm{~mA}$. Figure 6 shows the number of trial versus the current at $V_{\mathrm{ds}}=800 \mathrm{mV}$.

4.3. RF Response with Parasitic Capacitance of MMFET. From Section 3 we conclude that intrinsic device delay metric of CNTFET has high performance. However, from a circuit/system perspective, a realistic scenario should incorporate all the necessary parasitics. For the sake of comparison, we have studied the MMFET device with same characteristics as Si MOSFETs of the $65 \mathrm{~nm}$ technology. The parasitic capacitance per nanotube $C_{\text {tube }}\left(C_{\text {tube }}\right.$ is the capacitance added when a one nanotube is added to our device) between the gate and the source (drain) electrode is computed using Fastcap [18]. The thickness of the Pd source (drain) film is

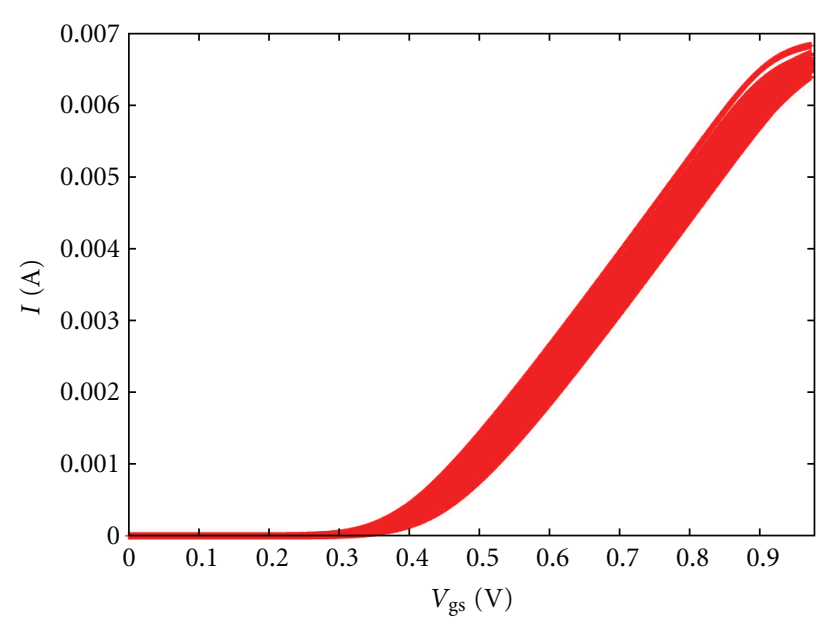

FIgURE 5: Impact of the diameter dispersion on the $I_{\mathrm{ds}}-V_{\mathrm{gs}}$ characteristic at $V_{\mathrm{ds}}=800 \mathrm{mV}$.

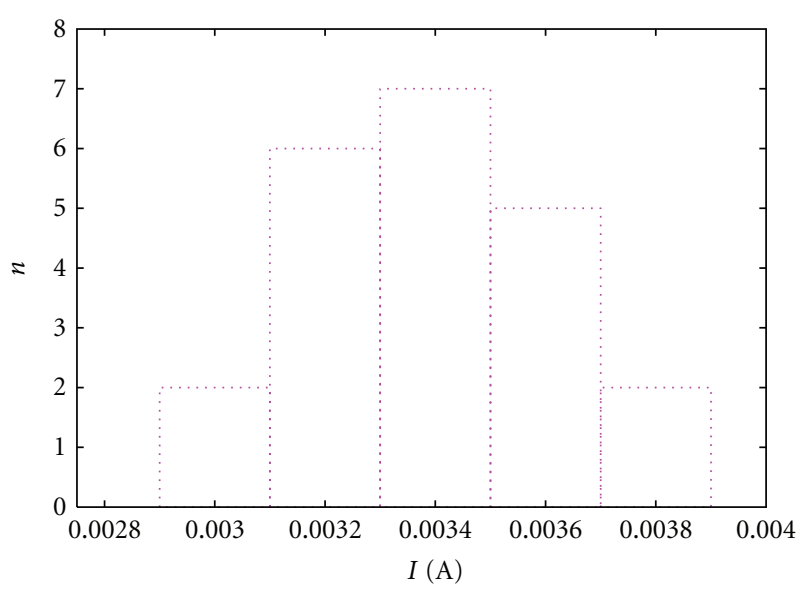

FIgURE 6: The number of trial versus the current at $V_{\mathrm{ds}}=800 \mathrm{mV}$.

$100 \mathrm{~nm}$ thick and $60 \mathrm{~nm}$ of length, the gate insulator $\mathrm{Al}_{2} \mathrm{O}_{3}$ thickness is $65 \mathrm{~nm}$ with dielectric constant $\sim 9$, and the $\mathrm{Al}$ gate is $150 \mathrm{~nm}$ thick and $140 \mathrm{~nm}$ length. It is assumed that each nanotube introduces a $4 \mathrm{~nm}$ width. Using Fastcap, with the geometries shown in Figure 7, we obtain $C_{\text {tube }} \sim 0.1 \mathrm{fF}$. In Figure 10 we show the discretization used in Fastcap for the geometries that we proposed. (For the sake of clearness we give details on the simulation of the capacitor by Fastcap:

(i) we generate two cuboid with cubegen;

(ii) the generated objects are combined in a list were we define their position with respect to each other and the dielectric material;

(iii) we run Fastcap on the lst file with the option $l$. To produce a picture of the capacitor the $m$ option should be used.)

In this simulation, we have used the measured parasitic capacitance for the multifinger device found in [10] as our 


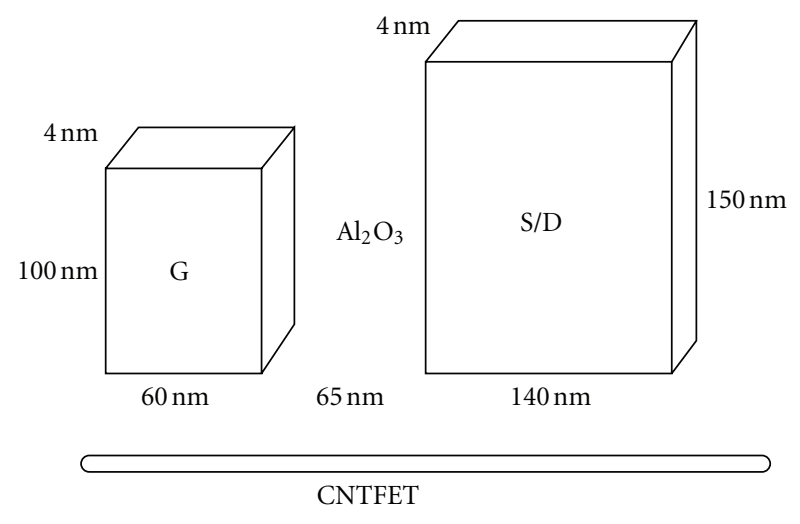

FIGURE 7: The used geometries for calculating the parasitic capacitance per nanotube $C_{\text {tube }}$ in the MMFET.

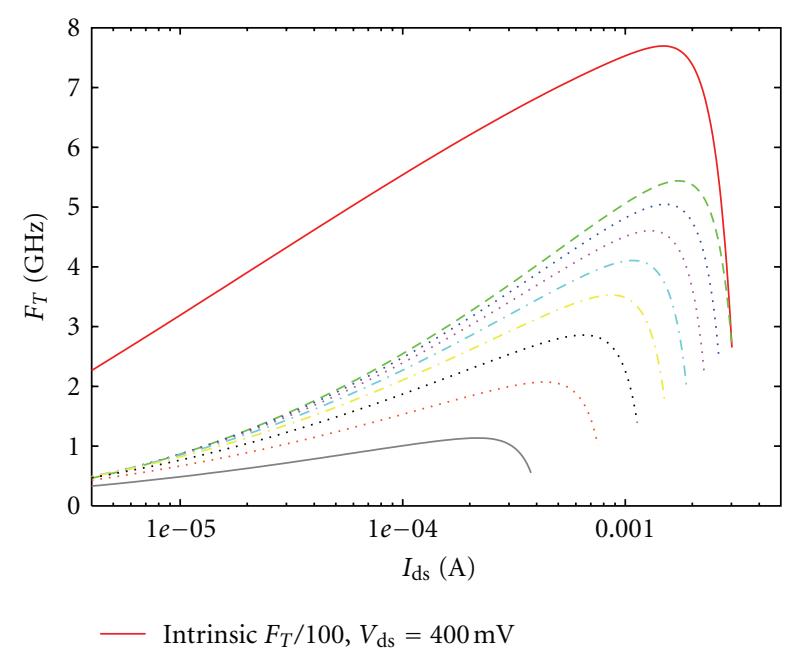

FIGURE 8: RF response with parasitic capacitance of MMFET at $V_{\mathrm{ds}}=400 \mathrm{mV}$ : from top down the number of nanotube changes from 16 to 2 by step of 2 . Intrinsic $F_{T} / 100$ solid line. Same parameters as CMOS $65 \mathrm{~nm}$ technology are used (see text).

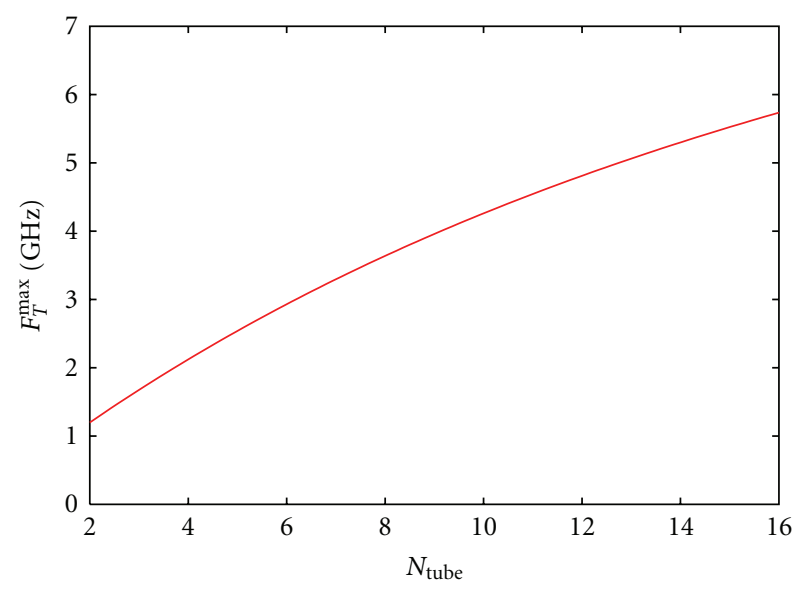

FIgure 9: The maximum value of extrinsic $F_{T}$ of the MMFET at $V_{\mathrm{ds}}=400 \mathrm{mV}$ versus number of nanotubes, $n_{\text {tube }}$.

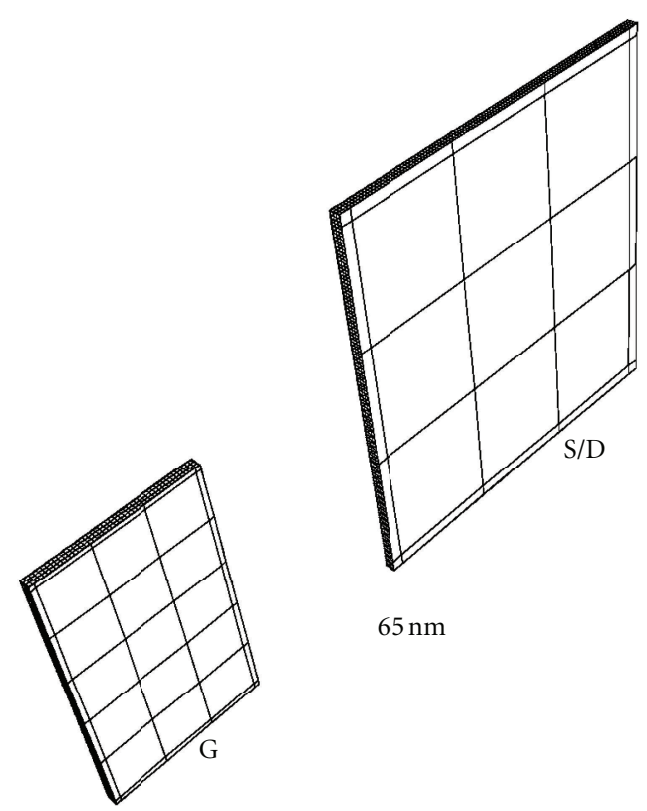

Figure 10: Discretization used in Fastcap.

initial value $C_{\exp } \sim 50 \mathrm{fF}$, and for each nanotube we have introduced $C_{\text {tube }}$ in such way:

$$
C_{\text {total }}=C_{\text {exp }}+\left(N_{\text {tube }}-1\right) N_{\text {finger }} C_{\text {tube }},
$$

where $N_{\text {tube }}$ and $N_{\text {finger }}$ are the number of tubes and fingers in the device, respectively. (With a new technology this value can be reduced significantly.)

In Figure 8, we show the results of simulation based on our model for the $F_{T}$ as function of $I_{\mathrm{ds}}$ for $V_{\mathrm{ds}}=400 \mathrm{mV}$ : the intrinsic $F_{T} / 100$ (solid line), and from top down extrinsic MMFET where the number of nanotube change from 16 to 2 by step of 2 . The device parameters used in this simulation are summarized in Table 2. As expected, the MMFET device that we propose improves the performance of the carbon nanotube in the RF regime where we found the maximum extrinsic $F_{T}^{\text {ex }} \sim 6 \mathrm{GHz}$ (see Figure 9) and the maximum intrinsic $F_{T}^{\text {in }} \sim 800 \mathrm{GHz}$ value that has to be compared to the maximal measured value $F_{T}^{\text {in }} \sim 10.2 \mathrm{GHz}[9]$.

4.4. Ring Oscillator with Carbon Nanotube. The parasitic capacitors play important role in the delay characteristics of a MMFET-based circuit. Simulations have been done with a three-stage ring oscillator (see Figures 11 and 12) and the effect of these parasitics has been studied. Figure 13 shows the effect on the natural oscillation frequency of parasitic capacitors with the parameters summarized in Table 2. (Of course the parasitic capacitance is not taken from Table 2.) It can be concluded from Figure 13 that in the range studied here, the parasitics dominate the performance. It should be noted, however, that the accurate estimation of the parasitics would be possible only when the layout geometry and a fabrication process of the MMFETs will be known. Further, it is worthwhile to mention that, for long channel MMFET under high bias, the effects of scattering with phonon are important and have to be considered. 
TABLE 2: CNTFET parameters.

\begin{tabular}{llcc}
\hline Parameter & Name & Value & Unit \\
\hline$n$ & Helicity parameter & 19 & \\
$m$ & Helicity parameter & 0 & \\
$d$ & Diameter & $1.49 \times 10^{-9}$ & $\mathrm{~m}$ \\
$T$ & Temperature & 300 & $\mathrm{~K}$ \\
$R_{d}$ & Drain contact resistance & $17 \times 10^{3}$ & $\Omega$ \\
$R_{s}$ & Source contact resistance & $23 \times 10^{3}$ & $\Omega$ \\
$R_{g}$ & Gate contact resistance & 10 & $\Omega$ \\
$L$ & Nanotube length & $8250 \times 10^{-9}$ & $\mathrm{~m}$ \\
$V_{\mathrm{FB}}$ & Flat band potential & $-40 \times 10^{-3}$ & $\mathrm{~V}$ \\
$C_{I}$ & Gate oxide capacitance & $100 \times 10^{-12}$ & $\mathrm{~F} / \mathrm{m}$ \\
$C_{\mathrm{DE}}$ & Drain capacitance & $0.1 \times 10^{-18}$ & $\mathrm{~F}$ \\
$C_{\mathrm{SE}}$ & Source capacitance & $0.1 \times 10^{-18}$ & $\mathrm{~F}$ \\
$C_{\mathrm{GS}}$ & Gate-source capacitance & $87.5 \times 10^{-15}$ & $\mathrm{~F}$ \\
$C_{\mathrm{GD}}$ & Gate-drain capacitance & $87.5 \times 10^{-15}$ & $\mathrm{~F}$ \\
$n_{\text {tube }}$ & Number of tubes & 16 & - \\
$n_{\mathrm{finger}}$ & Number of finger & 25 & - \\
\hline
\end{tabular}

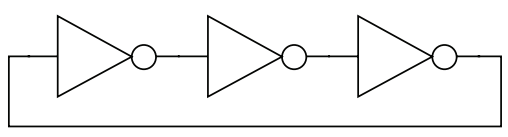

FiguRE 11: Three-stage ring oscillator.

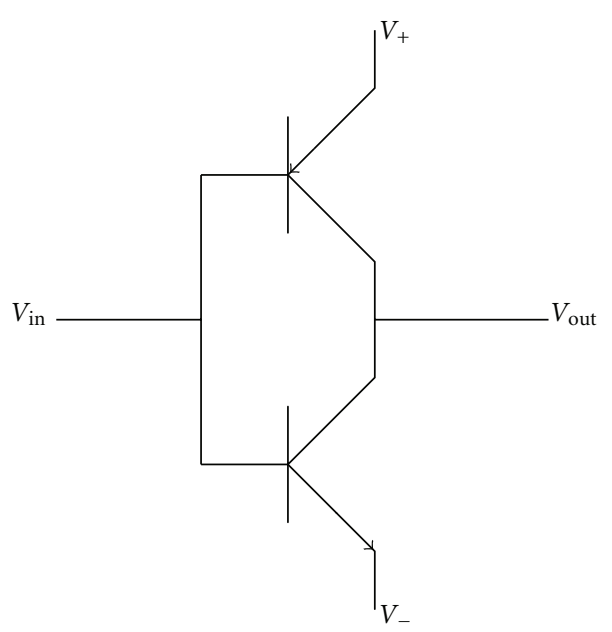

FIGURE 12: Schematic representation of a single inverter with $V_{+}=$ $0.4 \mathrm{~V}$ and $V_{-}=0.4 \mathrm{~V}$.

\section{Conclusions}

This paper use circuit-compatible modeling technique for the ballistic CNTFET for applications to radio frequency circuits. Results of simulation for radio frequency circuits using VHDL-AMS simulator in the intrinsic case are presented. A new architecture was proposed to improve the performance of the CNTFET. We found that the extrinsic $F_{T}$ and the natural oscillation frequency of a three-stage ring oscillator are $\sim 10 \mathrm{Ghz}$ in the cases considered here. An experimental

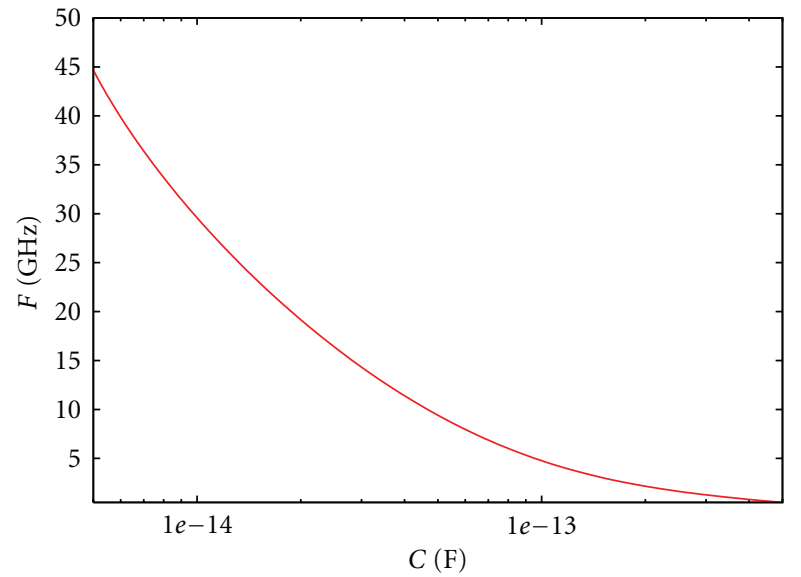

FIGURE 13: Natural oscillation frequency as function of $C_{\text {para }}$ under ballistic transport.

validation for the obtained results is necessary. From our analysis on the high-frequency performance of CNTFET, we conclude the following.

(i) Classical lithography reproduces the same problem as CMOS. To overcome this problem we propose a metallic nanotube contacts.

(ii) Effect of phonon scattering can be important and have to be considered in the RF regime.

(iii) Non-quasistatic effects may not be very important.

We hope to address some of these issues in the near future.

\section{Acknowledgment}

The authors would like to thank the support of this work by the Institut TELECOM under NanoRF project.

\section{References}

[1] P. L. McEuen, M. S. Fuhrer, and H. Park, "Single-walled carbon nanotube electronics," IEEE Transactions on Nanotechnology, vol. 1, no. 1, pp. 78-85, 2002.

[2] S. J. Tans, A. R. M. Verschueren, and C. Dekker, "Roomtemperature transistor based on a single carbon nanotube," Nature, vol. 393, no. 6680, pp. 49-52, 1998.

[3] R. Martel, T. Schmidt, H. R. Shea, T. Hertel, and P. Avouris, "Single- and multi-wall carbon nanotube field-effect transistors," Applied Physics Letters, vol. 73, no. 17, pp. 2447-2449, 1998.

[4] M. P. Anantram and F. Léonard, "Physics of carbon nanotube electronic devices," Reports on Progress in Physics, vol. 69, no. 3, pp. 507-561, 2006.

[5] S. J. Wind, J. Appenzeller, and P. Avouris, "Vertical scaling of carbon nanotube field-effect transistors using top gate electrodes," Applied Physics Letters, vol. 80, no. 20, p. 3817, 2002.

[6] A. Javey, J. Guo, Q. Wang, M. Lundstrom, and H. Dai, "Ballistic carbon nanotube field-effect transistors," Nature, vol. 424, no. 6949, pp. 654-657, 2003. 
[7] A. Javey, J. Guo, D. B. Farmer et al., "Self-aligned ballistic molecular transistors and electrically parallel nanotube arrays," Nano Letters, vol. 4, no. 7, pp. 1319-1322, 2004.

[8] Y.-M. Lin, J. Appenzeller, Z. Chen, Z.-G. Chen, H.-M. Cheng, and P. Avouris, "High-performance dual-gate carbon nanotube FETs with 40-nm gate length," IEEE Electron Device Letters, vol. 26, no. 11, pp. 823-825, 2005.

[9] K. Narita, H. Hongo, M. Ishida, and F. Nihey, "RF performance of multiple-channel carbon nanotube transistors," in Proceedings of the Trends in NanoTechnology Conference (TNT '06), Grenoble, France, September 2006.

[10] D. Wang, Z. Yu, S. McKernan, and P. J. Burke, "Ultrahigh frequency carbon nanotube transistor based on a single nanotube," IEEE Transactions on Nanotechnology, vol. 6, no. 4, pp. 400-403, 2007.

[11] J. Guo, S. Datta, and M. Lundstrom, "A numerical study of scaling issues for schottky barrier carbon nanotube transistors," Condensed Matter, vol. 1, 26 pages, 2003.

[12] J. Guo, M. Lundstrom, and S. Datta, "Performance projections for ballistic carbon nanotube field-effect transistors," Applied Physics Letters, vol. 80, no. 17, pp. 3192-3194, 2002.

[13] A. Raychowdhury, S. Mukhopadhyay, and K. Roy, "A circuitcompatible model of ballistic carbon nanotube field-effect transistors," IEEE Transactions on Computer, vol. 23, no. 10, pp. 1411-1420, 2004.

[14] C. Maneux, J. Goguet, S. Fregonese, T. Zimmer, H. Cazin d'Honincthun, and S. Galdin-Retailleau, "Analysis of CNTFET physical compact model," in Proceedings of the IEEE International Conference on Design and Test of Integrated Systems in Nanoscale Technology (DTIS '06), pp. 40-45, La Marsa, Tunisia, September 2006.

[15] P. Desgreys, J. G. da Silva, and D. Robert, "Dispersion Impact on Ballistic CNTFET n+-i-n+ Performances," in Proceedings of the European Nano Systems Worshop (ENS '06), Paris, France, December 2006.

[16] R. Saito, G. Dresselhaus, and M. S. Dresselhaus, Physical Properties of Carbon Nanotubes, Imperial College Press, London, UK, 1998.

[17] A. Keshavarzi, A. Raychowdhury, J. Kurtin, K. Roy, and V. De, "Carbon nanotube field-effect transistors for high-performance digital circuits-Transient analysis, parasitics, and scalability," IEEE Transactions on Electron Devices, vol. 53, no. 11, pp. 2718-2726, 2006.

[18] K. Nabors and J. White, "FastCap: a multipole accelerated 3-D capacitance extraction program," IEEE Transactions on Computer, vol. 10, no. 11, pp. 1447-1459, 1991. 

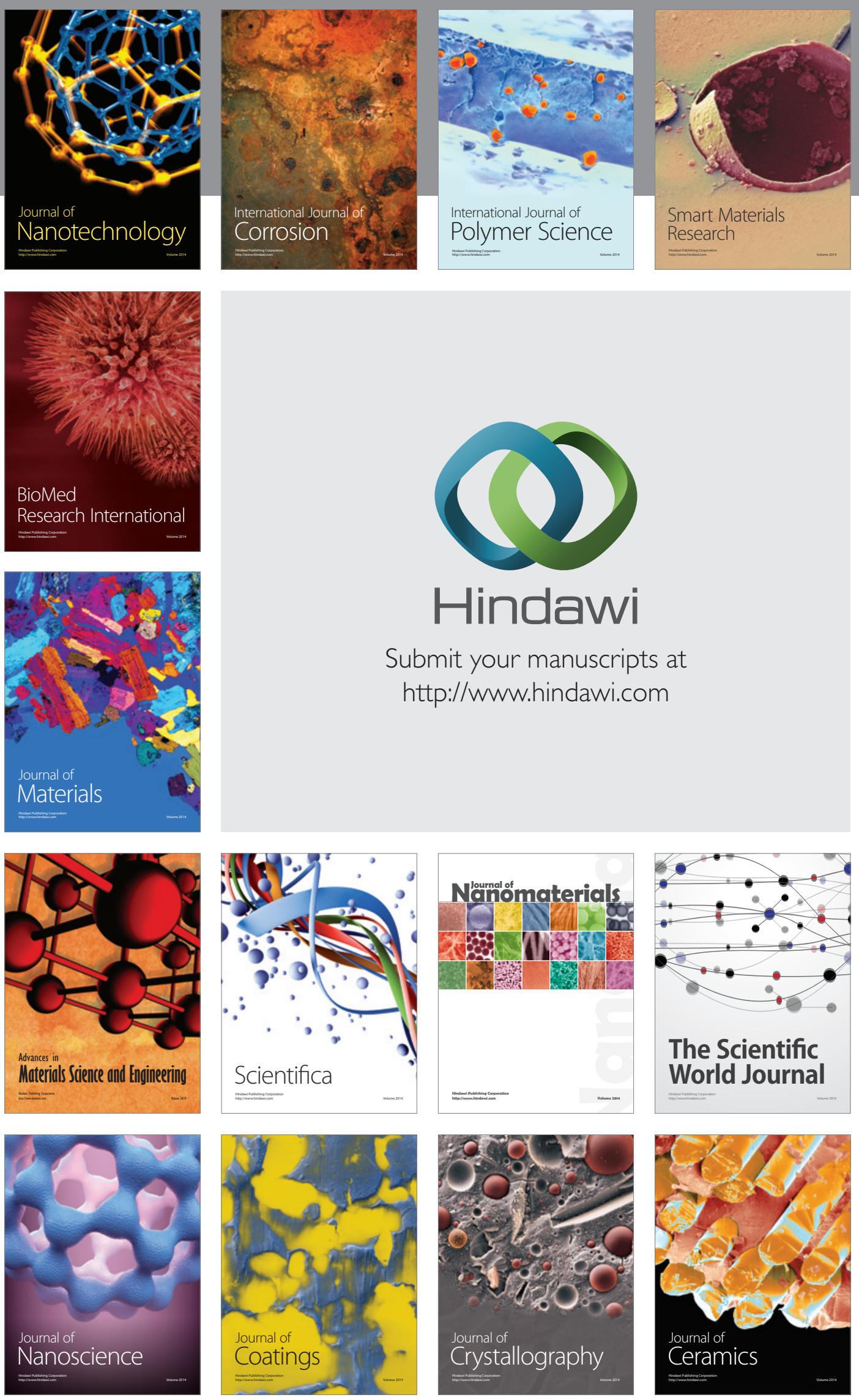

The Scientific World Journal

Submit your manuscripts at

http://www.hindawi.com

\section{World Journal}

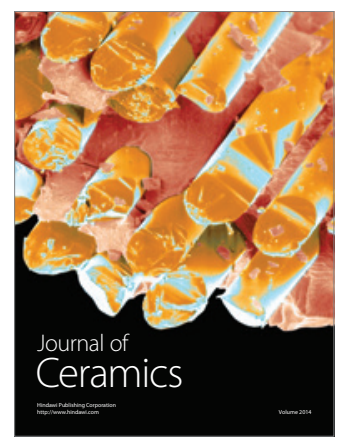

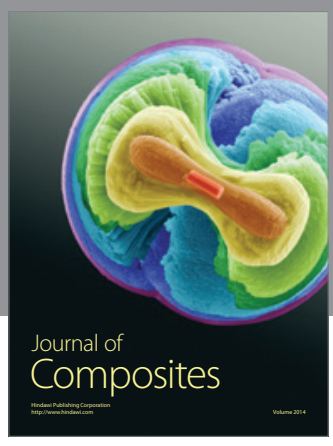
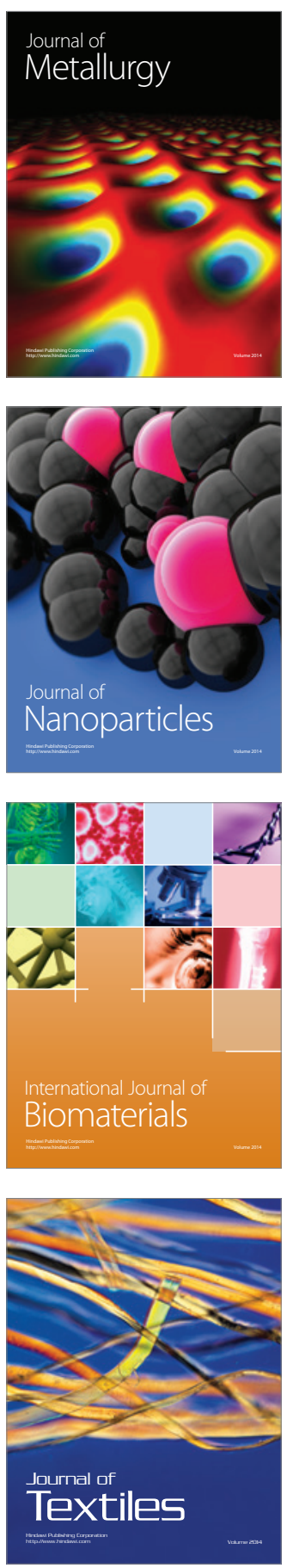